\title{
Origin of the enhanced flexoelectricity of relaxor ferroelectrics
}

\author{
Jackeline Narvaez ${ }^{1,2, a)}$ and Gustau Catalan ${ }^{1,3, b)}$ \\ ${ }^{1}$ ICN2-Institut Catala de Nanociencia i Nanotecnologia, Campus UAB, 08193 Bellaterra (Barcelona), Spain \\ ${ }^{2}$ CSIC - Consejo Superior de Investigaciones Cientificas, ICN2 Building, Campus UAB, 08193 Bellaterra \\ (Barcelona), Spain \\ ${ }^{3}$ ICREA - Institucio Catalana de Recerca i Estudis Avançats, 08010 Barcelona, Spain
}

(Received 21 February 2014; accepted 6 April 2014; published online 23 April 2014)

\begin{abstract}
We have measured the bending-induced polarization of $\mathrm{Pb}\left(\mathrm{Mg}_{1 / 3} \mathrm{Nb}_{2 / 3}\right) \mathrm{O}_{3}-\mathrm{PbTiO}_{3}$ single crystals with compositions at the relaxor-ferroelectric phase boundary. The crystals display flexocoupling coefficients $\mathrm{f}>100 \mathrm{~V}$, an order of magnitude bigger than the theoretical upper limit set by the theories of Kogan and Tagantsev. This enhancement persists in the paraphase up to a temperature $\mathrm{T}^{*}=500 \pm 25 \mathrm{~K}$ that coincides with the onset of anelastic softening in the crystals; above $\mathrm{T}^{*}$, the true (lattice-based) flexocoupling coefficient is measured as $f_{13} \approx 10 \mathrm{~V}$ for both compositions. Cross-correlation between flexoelectric, dielectric, and elastic properties indicates that the enhancement of bending-induced polarization of relaxor ferroelectrics is not caused by intrinsically giant flexoelectricity but by the reorientation of polar nanodomains that are ferroelastically active below T*. ㅇ 2014 AIP Publishing LLC. [http://dx.doi.org/10.1063/1.4871686]
\end{abstract}

The giant electromechanical performance of relaxorbased ferroelectrics ${ }^{1}$ and the complex physics associated with their inherently nanoscopic phase separation have inspired much research into these compounds. ${ }^{2}$ The archetypal relaxor, $\mathrm{Pb}\left(\mathrm{Mg}_{1 / 3} \mathrm{Nb}_{2 / 3}\right) \mathrm{O}_{3}$ (PMN), was also the first ceramic for which bending-induced polarization (flexoelectricity $)^{3,4}$ was ever measured, ${ }^{5}$ and it was its unexpectedly large value of the flexoelectric coefficient that triggered the investigation of flexoelectricity in other perovskite ferroelectrics such as $\mathrm{Pb}(\mathrm{Zr}, \mathrm{Ti}) \mathrm{O}_{3},{ }^{6,7} \mathrm{BaTiO}_{3}{ }^{8}$ and $(\mathrm{Ba}, \mathrm{Sr}) \mathrm{TiO}_{3},{ }^{9}$ these investigations, together with the realization that very large flexoelectric effects can be achieved in the nanoscale,${ }^{10-12}$ have contributed to the current surge of interest in this phenomenon. ${ }^{13}$

Yet, for all the research, we still do not know something as basic as the intrinsic value of the effective flexoelectric coefficients-the constants of proportionality between strain gradient and induced polarization. With the exception of $\mathrm{SrTiO}_{3},{ }^{14}$ for most perovskites, the experimentally measured flexoelectricity exceeds theoretical expectations by between one and three orders of magnitude. ${ }^{15-18}$ And differences are not merely between theory and experiment, experimental results can also substantially disagree among themselves: in $\mathrm{Pb}\left(\mathrm{Mg}_{1 / 3} \mathrm{Nb}_{2 / 3}\right) \mathrm{O}_{3}-10 \% \mathrm{PbTiO}_{3}$, for example, there is a discrepancy of three orders of magnitude between flexoelectric coefficients measured by two different methods. ${ }^{19}$ Meanwhile, the expected contribution of polar nanoregions to the flexoelectricity of relaxor ferroelectrics ${ }^{3}$ has not been established. Nor are there, surprisingly, any measurements for compositions at or near the morphotropic phase boundary, even though their otherwise record-high electromechanical performance ${ }^{1}$ might suggest the possibility of similarly enhanced flexoelectric effects. To further complicate the picture, most flexoelectric measurements to date have been performed in ceramics, and there are no experimental reports

\footnotetext{
${ }^{\text {a)} E l e c t r o n i c ~ m a i l: ~ j a c k e l i n e . n a r v a e z @ c i n 2 . e s ~}$

b)Electronic mail: gustau.catalan@
}

for single crystals other than $\mathrm{SrTiO}_{3} ;{ }^{14,20}$ this is relevant because grain boundaries have their own piezoelectric properties $^{21,22}$ that can add an extrinsic contribution to the bending-induced polarization.

In this context, we have studied the bending-induced polarization of single crystal relaxor-ferroelectrics with compositions $\quad(1-\mathrm{x}) \mathrm{Pb}\left(\mathrm{Mg}_{1 / 3} \mathrm{Nb}_{2 / 3}\right) \mathrm{O}_{3}-\mathrm{xPbTiO}_{3}$, with $\mathrm{x}=0.28$ and 0.34 (hereafter, labelled PMN-28\%PT and PMN-34\%PT). The dimensions are $6.58 \times 2.54 \times 0.5 \mathrm{~mm}$, and their surface is parallel to the $\{100\}_{\text {pseudocubic }}$ planes, with the edges parallel to the $\langle 100\rangle$ crystallographic axes. These crystals, commercially available (TRS Technologies, Inc.), are at the morphotropic boundary that separates a relaxor-like rhombohedral phase for PMN-rich compositions from a ferroelectric tetragonal phase for PT-rich compositions. ${ }^{23}$ We find that the flexocoupling voltage is indeed large, exceeding theoretical expectations ${ }^{13}$ by an order of magnitude.

Deformation-induced polarization may arise from extrinsic origins such as defect dipoles, built-in pyroelectricity or even microcracking, so careful analysis is required to clarify the origin of the observed enhancement. Close inspection of the temperature dependence revealed a direct correlation between the enhancement of apparent flexoelectricity and the onset of anelastic softening in the materials. The mechanical softening and enhanced flexoelectric response are both consistent with the onset of ferroelasticity within polar nanodomains at a temperature $\mathrm{T}^{*}$ higher than the dielectric peak. ${ }^{24,25}$ The "giant" bending-induced polarization of these relaxor ferroelectrics is thus not due to an intrinsic (latticebased) giant flexoelectricity, but to a bending-induced reorientation of polar nanodomains.

We measure the bending-induced polarization using the method described by Zubko et al.: ${ }^{14}$ a dynamic mechanical analyzer (DMA 8000, Perkin-Elmer) applies a periodic threepoint bending stress whilst simultaneously recording the elastic response (storage modulus and elastic loss). The DMA's mechanical force signal is fed into the reference channel of a lock-in amplifier (Stanford Research Instruments, model 
830), while the samples' electrodes are connected to the measurement channel of the lock-in amplifier, which records the bending-induced displacement currents. The displacement current is converted into polarization using $\mathrm{P}=\mathrm{I} /(2 \pi \nu \mathrm{A})$, where $\nu$ is the frequency of the applied force $(13 \mathrm{~Hz}$ in our experiments) and $\mathrm{A}$ is the area of the electrodes. The polarization measured by the lock-in is related to the effective flexoelectric coefficient $\mu_{13}{ }^{\text {eff }}$ by

$$
\overline{\mathrm{P}}_{3}=\mu_{13}{ }^{\text {eff }} \frac{\overline{\partial \epsilon_{11}}}{\partial \mathrm{x}_{3}} \quad \text { and } \quad \overline{\frac{\partial \epsilon_{11}}{\partial \mathrm{x}_{3}}}=\frac{12 \mathrm{z}_{0}}{\mathrm{~L}^{3}}(\mathrm{~L}-\mathrm{a}),
$$

where $L$ is the separation between the standing points of the crystal, $a$ is the half-length of the electrodes, and $\mathrm{z}_{0}$ is the displacement applied in the middle of the sample. Typical values in our measurements are $\mathrm{L}=5.40 \mathrm{~mm}, \mathrm{a}=2.7 \mathrm{~mm}$, and $\mathrm{z}_{0}=2 \mu \mathrm{m}$. The flexoelectric tensor components are always coupled together and cannot be individually measured in quasistatic bending experiments; ${ }^{20,26}$ it is customary instead to define an effective coefficient that is a combination of the tensor components relating the strain gradient to the induced polarization. In the three-point bending geometry of our experiment, the effective flexoelectric coefficient is $\mu_{13}{ }^{\text {eff }} \equiv \frac{\mathrm{P}_{3}}{\partial \mathrm{s}_{1} / \partial \mathrm{x}_{3}}$, where sample length is along $\mathrm{x}_{1}$, width along $\mathrm{x}_{2}$, and thickness along $\mathrm{x}_{3}$. For an isotropic material, $\mu_{13}{ }^{\text {eff }}$ is related to the flexoelectric tensor components by ${ }^{13,14,18}$ $\mu_{13}{ }^{\text {eff }}=\mu_{11}+(1-\nu) \mu_{13}$, where $\nu$ is the Poisson's ratio. The elastic and flexoelectric responses are recorded between room temperature and $573 \mathrm{~K}\left(300^{\circ} \mathrm{C}\right)$, both on heating and cooling, using a ramp-rate of $3 \mathrm{~K} / \mathrm{min}$.

Because flexoelectricity is proportional to dielectric permittivity, ${ }^{3,4,13,27}$ it is useful to characterize the dielectric constant of the crystals. The dielectric constant and loss as a function of temperature (Figure 1) were measured at $1 \mathrm{kHz}$ using an Agilent E4980A Precision LCR-meter to measure the capacitance and the DMA receptacle to control the

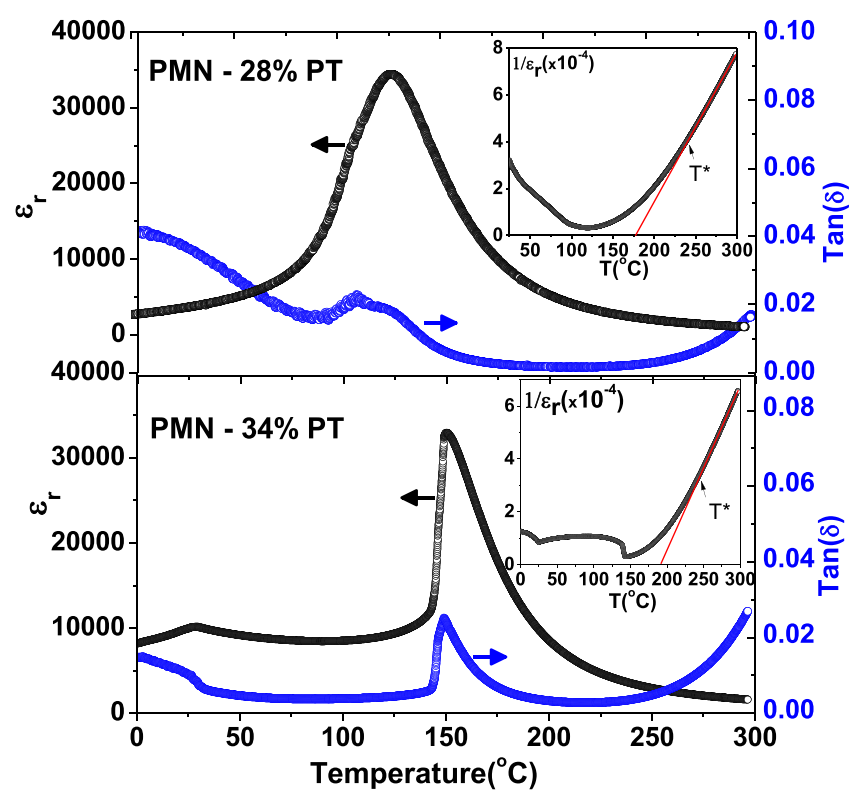

FIG. 1. Relative dielectric constant (black) and loss tangent (blue) of PMN-28\% PT and PMN-34\%PT. Insets: inverse of the relative permittivity, showing a departure from linear Curie-Weiss behaviour below $\mathrm{T}^{*}$. temperature. The heating and cooling ramps were identical to those of the flexoelectric measurements $(3 \mathrm{~K} / \mathrm{min})$. The dielectric losses are low $(\tan \delta<0.05)$ for both samples throughout the entire temperature range of the experiments: this ensures that the impedance response is predominantly dielectric even at the highest recorded temperatures, where dielectric losses start to rise due to increased conductivity.

Both samples display clear dielectric maxima, but with differences: the peak of PMN-34\%PT is sharper and at higher temperature than that of PMN-28\%PT. This is to be expected and correlates with the concentration of $\mathrm{PbTiO}_{3}$ (PT), which is a standard ferroelectric with a high Curie temperature $\left(\mathrm{T}_{\mathrm{C}}=492^{\circ} \mathrm{C}\right):{ }^{28}$ PMN-34\% PT has a dielectric response closer to that of standard ferroelectric $\mathrm{PbTiO}_{3}$ (sharp peak and a higher Curie temperature $\mathrm{T}_{\mathrm{C}}=150^{\circ} \mathrm{C}$ ) while the PMN-28\%PT sample has response closer to that of conventional relaxor $\mathrm{Pb}\left(\mathrm{Mn}_{1 / 2} \mathrm{Nb}_{2 / 3}\right) \mathrm{O}_{3}$, with increased diffuseness and broad maximum at a lower temperature $\left(\mathrm{Tm}=125^{\circ} \mathrm{C}\right)$. Notice that, though PMN-34\%PT is more ferroelectric-like and PMN-28\% more relaxor-like, the inverse permittivity (inset of Figure 1) departs from linear Curie-Weiss behaviour below $\mathrm{T}^{*} \sim 250^{\circ} \mathrm{C}$ for both compositions. Such departure is a classic indicator of the existence of polar nanoregions characteristic of relaxors. ${ }^{29}$ Thus, irrespective of whether the transition is diffuse or sharp, the high temperature phase is relaxor-like for both PMN-28\%PT and PMN-34\%PT. Transitions from a high temperature relaxor-like phase to a long-range ferroelectric phase are typical for compositions at the boundary between the two states. $^{2}$

The effective flexoelectric coefficients as a function of temperature are shown in Figure 2, together with the simultaneously measured Young's modulus and elastic loss tangent. Flexoelectricity and permittivity peak at the same temperature, with flexoelectric maxima of $30-40 \mu \mathrm{C} / \mathrm{m}$. There are no other single crystal values in the literature, but for ceramics of pure PMN (Ref. 5) the flexoelectric maximum is $8 \mu \mathrm{C} / \mathrm{m}$. Though the $5 \times$ bigger flexoelectricity of PMN-PT may be due to different sample morphology between crystals and ceramics, we think this is unlikely (permittivity, for example, differs only by a factor of $<2$ ), so it seems that closeness to the morphotropic phase boundary may indeed lead to bigger effective flexoelectric coefficients, though still below those of barium titanate-based solid solutions. ${ }^{9,13,18,30}$ Very importantly, however, the large flexoelectric coefficients are dependent on the thermal history of the sample: up to $\mathrm{T}^{*} \sim 225-250^{\circ} \mathrm{C}$, flexoelectricity is higher on heating than on cooling, suggesting a role of domains. The difference is more pronounced in PMN-28\%PT, which is consistent with a bigger fraction metastable domains in the more relaxorlike compound.

It is useful to also examine the elastic behaviour in Figure 2. At high temperatures, the Young's modulus $E$ is relatively constant, but around $200-250^{\circ} \mathrm{C}$ the lattice begins to soften, with the Young's modulus decreasing from $60 \mathrm{GPa}$ above $\mathrm{T}^{*}$ to $20 \mathrm{GPa}$ below $\mathrm{T}_{\mathrm{m}}$ for PMN-28\% $\mathrm{PT}$, and from $80 \mathrm{GPa}$ to $40 \mathrm{GPa}$ for PMN-34\%PT. Relaxor ferroelectrics are known to display a peak in acoustic emission at a relatively composition-independent temperature $\mathrm{T}^{*} \approx 500 \pm 30 \mathrm{~K} ;{ }^{24,25}$ since acoustic emission is caused by a sudden release of 


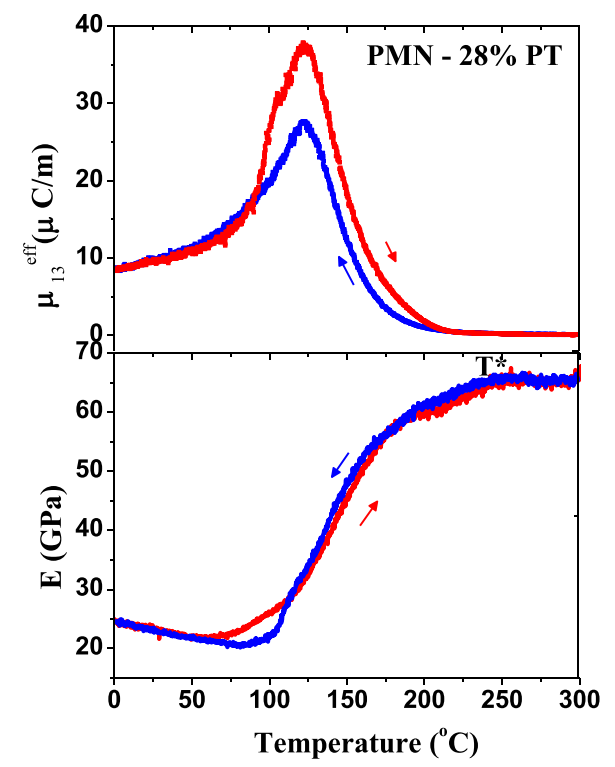

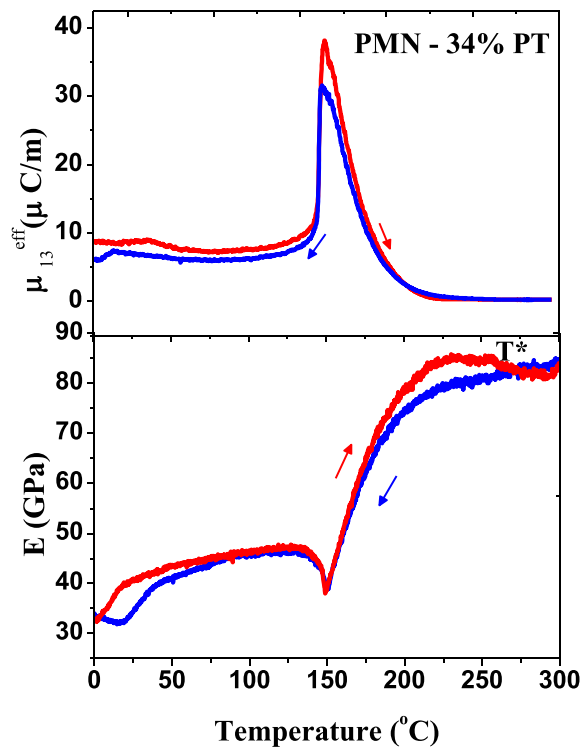

FIG. 2. Temperature dependence of the flexoelectric coefficients and elastic Young's modulus of PMN-28\%PT and PMN-34\%PT. elastic energy, $\mathrm{T}^{*}$ must signal an elastic discontinuity, in agreement with our results and also with analysis by resonant ultrasound spectroscopy. ${ }^{31}$ The origin of the peak in acoustic emission and of mechanical anelastic softening below $\mathrm{T}^{*}$ is attributed to a ferroelastic transition within the relaxor polar nanoregions. These regions appear at a higher so-called Burns temperature $\left(\mathrm{T}_{\mathrm{b}} \geq 600 \mathrm{~K}\right.$ (Ref. 25)) but only become ferroelastically active at $\mathrm{T}^{*} \approx 500 \mathrm{~K} .^{24,25,31,32}$ Further evidence of the role of ferroelastic nanodomains is provided by the thermal history dependence of the bending-induced polarization, which is higher on heating than on cooling, consistent with a bigger volume fraction of nanodomains when heating from the ferroelectric polar state than when cooling from the non-polar paraphase.

In order to gain further insight into the high values of flexoelectricity, we examine the flexoelectric coefficient normalized by the dielectric constant (Figure 3). Theoretically, this flexo-coupling (or flexo-voltage ${ }^{12}$ ) coefficient $f$ should be of the order of $1-10 \mathrm{~V} .^{13}$ Experimentally, instead, the coefficients reach up to $f \sim 100-300 \mathrm{~V}$, a full order of magnitude bigger than the theoretical upper limit. At low temperatures, some or most of the bending-induced polarization may be attributed to piezoelectricity. In PMN-34\% PT, long range polarization appears at $\mathrm{T}_{\mathrm{C}}=\mathrm{Tm}=150{ }^{\circ} \mathrm{C}$. In PMN-28\%PT, which is more relaxor-like, spontaneous long range order does not appear at a critical temperature, and the flexocoupling coefficient grows continuously upon cooling. On heating, though, a residual ferroelectric anomaly appears around $\mathrm{T}_{\mathrm{C}}=105^{\circ} \mathrm{C}<\mathrm{Tm}=125^{\circ} \mathrm{C}$. Meanwhile, at temperatures above $\mathrm{T}^{*}$, the flexocoupling coefficient decreases to a stable value $f \leq 10 \mathrm{~V}$ that is not hysteretic, is constant with temperature, and is consistent with theoretical expectations. This is therefore likely to be the true intrinsic value of the flexocoupling coefficient.

The appearance and subsequent growth of nanotwins below $\mathrm{T}^{*}$ have profound consequences for the electromechanical response of the material, because now external stress can cause a ferroelastic reorientation of the nanodomains. Under bending stress, local compression of the $x-y$ plane at the concave side will increase the proportion of domains with perpendicular polarization. The bending strains reach a maximum of $\epsilon_{11} \approx$ (near the centre of curvature) at the surface; multiplied by the Young's modulus of PMN-PT (20-80 GPa), this is equivalent to a stress of 2-8 MPa, which is sufficient to cause ferroelastic switching ${ }^{33}$ and, combined with a small electric bias, it can pole PMN$\mathrm{PT}$ even at room temperature. ${ }^{33} \mathrm{We}$ emphasize, however, that an electric bias is still necessary in order to remove the degeneracy between the $+\mathrm{z}$ and $-\mathrm{z}$ directions: ${ }^{34}$ though stress can rearrange ferroelastic domains and locally favour vertical polarization, the electrostrictive coupling is to the square of polarization and thus cannot favour one sign over its opposite. ${ }^{12,13}$ This is the reason why mechanically assisted poling is always done in the presence of an electric bias. ${ }^{33}$ Since we are not applying any external voltage in our experiments, the biasing is likely to be provided by the

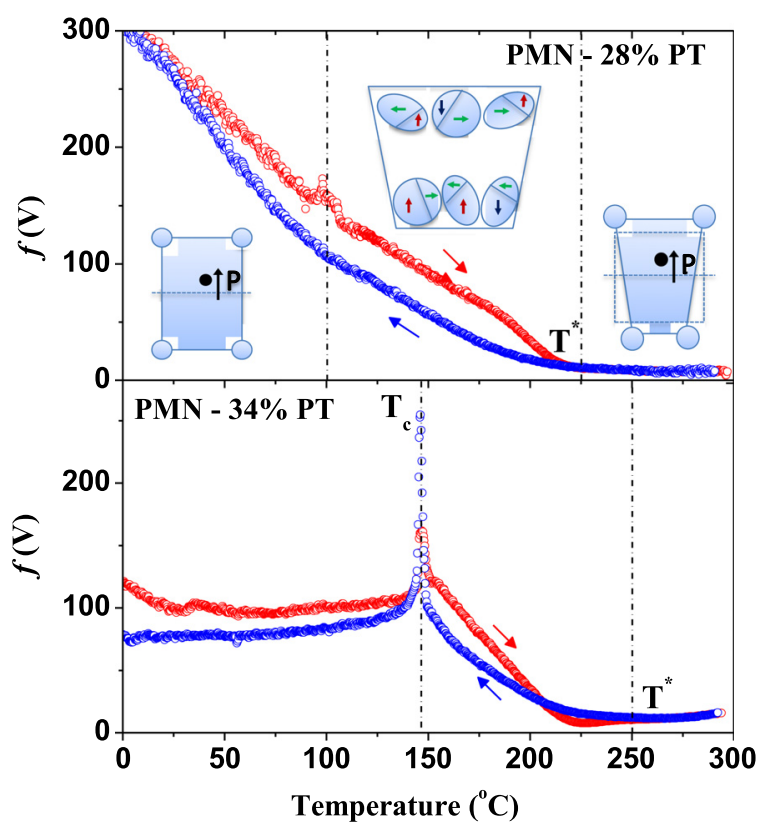

FIG. 3. Flexocoupling coefficients of PMN-28\%PT and PMN-34\%PT. 
flexoelectric field. The average strain gradient in the bent crystals is of the order of $\frac{\overline{\partial \epsilon_{11}}}{\partial z} \approx 0.2 \mathrm{~m}^{-1}$, and the intrinsic (high temperature) flexocoupling coefficient is of the order of $\mathrm{f} \approx 10 \mathrm{~V}$, so the equivalent flexoelectric field is of the order of $E=f \frac{\overline{\partial \epsilon_{11}}}{\partial z} \approx 2 \mathrm{~V} / \mathrm{m}$. By itself, this flexoelectric field would be too small to cause ferroelectric switching (typical coercive fields are of the order of $\mathrm{KV} / \mathrm{cm}$ ), but it is sufficient to break the inversion symmetry so that, as the stress causes ferroelastic switching, the strain gradient "loads the dice" in the poling direction.

Ferroelasticity and flexoelectricity must thus work in tandem to achieve the enhancement. This cooperative "flexoferroelastic" polarization is not bound by the KoganTagantsev limit and can therefore yield domain-based effective flexocoupling coefficients orders of magnitude larger than the intrinsic lattice-based flexoelectricity, which we have measured as a constant $f_{13}=10 \mathrm{~V}$ above $\mathrm{T}^{*}$. Though the potential usefulness of giant flexoelectricity for electromechanical transduction is mostly unaffected by its origin, the small dependence on thermal history, typical of domain-based properties, is undesirable and should be minimized. Perhaps more importantly, these results are evidence that giant bending-induced polarization can be obtained without an intrinsically giant flexoelectricity, this should help reconcile some of the present discrepancies about the true magnitude of the flexoelectric coefficient.

This work was funded by an ERC Starting Grant from the EU (Project No. 308023) and by Project MAT201017771 from the Spanish Ministry of Education. We are grateful to Dr. Neus Domingo for useful discussions in the preparation of this article.

${ }^{1}$ S. E. Park and T. R. Shrout, J. Appl. Phys. 82, 1804-1811 (1997).

${ }^{2}$ A. A. Bokov and Z.-G. Ye, J. Mater. Sci. 41, 31-52 (2006).

${ }^{3}$ S. M. Kogan, Sov. Phys. Solid State 5, 2069-2070 (1964).

${ }^{4}$ A. K. Tagantsev, Phys. Rev. B 34, 5883-5888 (1986).

${ }^{5}$ W. Ma and L. E. Cross, Appl. Phys. Lett. 78, 2920-2921 (2001).

${ }^{6}$ W. Ma and L. E. Cross, Appl. Phys. Lett. 82, 3293-3295 (2003).
${ }^{7}$ W. Ma and L. E. Cross, Appl. Phys. Lett. 86, 072905 (2005).

${ }^{8}$ W. Ma and L. E. Cross, Appl. Phys. Lett. 88, 232902 (2006).

${ }^{9}$ W. Ma and L. E. Cross, Appl. Phys. Lett. 81, 3440-3442 (2002).

${ }^{10}$ G. Catalan, L. J. Sinnamon, and J. M. Gregg, J. Phys.: Condens. Matter 16, 2253-2264 (2004).

${ }^{11}$ G. Catalan, A. Lubk, A. H. G. Vlooswijk, E. Snoeck, C. Magen, A. Janssens, G. Rispens, G. Rijnders, D. H. A. Blank, and B. Noheda, Nat. Mater. 10, 963-967 (2011).

${ }^{12} \mathrm{H}$. Lu, C.-W. Bark, D. Esque de los Ojos, J. Alcala, C. B. Eom, G. Catalan, and A. Gruverman, Science 336, 59-61 (2012).

${ }^{13}$ P. Zubko, G. Catalan, and A. K. Tagantsev, Annu. Rev. Mater. Res. 43, 387-421 (2013).

${ }^{14}$ P. Zubko, G. Catalan, A. Buckley, P. R. L. Welche, and J. F. Scott, Phys. Rev. Lett. 99, 167601 (2007).

${ }^{15}$ R. Maranganti and P. Sharma, Phys. Rev. B 80, 054109 (2009).

${ }^{16}$ J. Hong, G. Catalan, J. F. Scott, and E. Artacho, J. Phys.: Condens. Matter 22, 112201 (2010).

${ }^{17}$ J. Hong and D. Vanderbilt, Phys. Rev. B 88, 174107 (2013).

${ }^{18}$ L. Shu, X. Wei, L. Jin, Y. Li, H. Wang, and X. Yao, Appl. Phys. Lett. 102, 152904 (2013).

${ }^{19}$ P. Hana, Ferroelectrics 351, 196-203 (2007).

${ }^{20}$ P. Zubko, G. Catalan, A. Buckley, P. R. L. Welche, and J. F. Scott, Phys. Rev. Lett. 100, 199906 (2008).

${ }^{21}$ A. Kholkin, I. Bdikin, T. Ostapchuk, and J. Petzelt, Appl. Phys. Lett. 93, 222905 (2008).

${ }^{22}$ J. Petzelt, T. Ostapchuk, I. Gregora, I. Rychetský, S. Hoffmann-Eifert, A. V. Pronin, Y. Yuzyuk, B. P. Gorshunov, S. Kamba, V. Bovtun et al., Phys. Rev. B 64, 184111 (2001).

${ }^{23}$ B. Noheda, D. E. Cox, G. Shirane, J. Gao, and Z. G. Ye, Phys. Rev. B 66, 054104 (2002).

${ }^{24}$ M. Roth, E. Mojaev, E. Dul'kin, P. Gemeiner, and B. Dkhil, Phys. Rev. Lett. 98, 265701 (2007).

${ }^{25}$ B. Dkhil, P. Gemeiner, A. Al-Barakaty, L. Bellaiche, E. Dul'kin, E. Mojaev, and M. Roth, Phys. Rev. B 80, 064103 (2009).

${ }^{26}$ M. Stengel, Phys. Rev. B 88, 174106 (2013).

${ }^{27}$ E. V. Bursian and O. I. Zaikovskii, Sov. Phys. Solid State 10, 1121-1124 (1968).

${ }^{28}$ M. J. Haun, E. Furman, S. J. Jang, H. A. McKinstry, and L. E. Cross, J. Appl. Phys. 62, 3331-3338 (1987).

${ }^{29}$ D. Viehland, S. J. Jang, L. E. Cross, and M. Wuttig, Phys. Rev. B 46, 8003-8006 (1992).

${ }^{30}$ L. E. Cross, J. Mater. Sci. 41, 53-63 (2006).

${ }^{31}$ M. A. Carpenter, J. F. J. Bryson, G. Catalan, S. J. Zhang, and N. J. Donnelly, J. Phys.: Condens. Matter 24, 045902 (2012).

${ }^{32}$ M. A. Carpenter, J. F. J. Bryson, G. Catalan, and C. J. Howard, J. Phys.: Condens. Matter 24, 045901 (2012).

${ }^{33}$ A. E. McLaughlin, T. Liu, and S. C. Lynch, Acta Mater. 53, 4001-4008 (2005).

${ }^{34}$ B. Jaffe, W. R. Cook, and H. Jaffe, Piezoelectric Ceramics (Academic Press, London-New York, 1971). 